Short Communication

\title{
Application of hydroxyapatite/collagen composite material for maxillary sinus floor augmentation
}

\author{
Seigo Ohba ${ }^{1,2)}$, Rena Shido ${ }^{1)}$, and Izumi Asahina ${ }^{1,2)}$ \\ Department of Regenerative Oral Surgery, Institute of Biomedical Sciences, Nagasaki University, Nagasaki, Japan \\ ${ }^{2}$ Center for Oral and Maxillofacial Implants, Nagasaki University Hospital, Nagasaki, Japan
}

(Received April 4, 2021; Accepted May 24, 2021)

\begin{abstract}
The aim of this study was to clarify whether hydroxyapatite/ collagen composite material $(\mathrm{HAp} / \mathrm{Col})$ could be useful as a graft material for maxillary sinus floor augmentation (MSFA). MSFA and implant placement were performed simultaneously. When the lateral approach was employed, 3 out of 19 implants failed in 3 maxillary sinuses (success rate; $84.2 \%$ ), and in these cases the alveolar bone heights, cortical bone thicknesses and values of the implant stability quotient were smaller. If alveolar the bone height, cortical bone thickness, and healing period are optimized, $\mathrm{HAp} / \mathrm{Col}$ can be a useful graft material for MSFA.

Keywords; alveolar bone height, cortical bone thickness, hydroxyapatite/ collagen composite material, maxillary sinus floor augmentation
\end{abstract}

\section{Introduction}

Although autologous bone grafting is still the gold standard for bone augmentation - including maxillary sinus floor augmentation (MSFA) because of its osteogenicity, donor site morbidity is unavoidable. Therefore, to minimize the degree of surgical invention, several materials have been developed as alternatives to autologous bone. Jambhekar et al. [1] have reported that alloplastic material for bone augmentation markedly induced vital bone. However, some alloplastic materials remain in situ over a long period, and may influence the outcome of dental implant treatment, with a potential to cause infection [2]. Ideally, therefore, any alloplastic material should induce sufficient bone formation and be remodeled and entirely replaced with host bone.

Hydroxyapatite/collagen composite (HAp/Col) (ReFit, HOYA Technosurgical, Tokyo, Japan) is made up of $80 \% \mathrm{HA}$ and $20 \%$ collagen, and can be completely replaced with native bone. Its pore size is appropriate for cell migration into the material [2]. Moreover, $\mathrm{HAp} / \mathrm{Col}$ is highly porous (95\% porosity) and shows sponge-like characteristics under wet conditions, allowing close contact with surrounding bone within the bone defect. Accordingly, $\mathrm{HAp} / \mathrm{Col}$ is expected to facilitate early bone formation with good handleability. In fact, the previous study has shown that $\mathrm{HAp} / \mathrm{Col}$ preserved alveolar bone volume in tooth extraction sockets and disappeared completely within 3 months after surgery [3].

The aim of the present study was to clarify whether the indications for HAp/Col could be expanded to MSFA. As this was a first attempt at HAp/ Col use for this purpose, its safety and efficacy for MSFA were assessed.

\section{Materials and Methods}

This study was reviewed and approved by the Nagasaki University Hospital Clinical Research Ethics Committee (15102603) and was also registered in the University Hospital Medical Information Network Center (UMIN000027566).

Correspondence to Dr. Seigo Ohba, Department of Regenerative Oral Surgery, Nagasaki University, 1-7-1 Sakamoto, Nagasaki 852-8588, Japan

Fax: +81958197705 E-mail: sohba@nagasaki-u.ac.jp

Color figures can be viewed in the online issue at J-STAGE.

doi.org/10.2334/josnusd.21-0163

DN/JST.JSTAGE/josnusd/21-0163

\section{Patient inclusion criteria}

1) Healthy adult individuals (more than 20 years of age) seeking dental implant treatment with MSFA for missing maxillary molar teeth.

2) Provision of written informed consent to participate.

\section{Patient exclusion criteria}

1) Any individuals with uncontrolled diabetes, hypertension, hepatic or renal disorders.

2) Pregnant or lactating females.

3) Patients who were considered unsuitable for inclusion by the head of the study.

Endpoints

1) Primary endpoint: The proportion of implants surviving until final restoration.

2) Secondary endpoints:

(1) The value of the implant stability quotient (ISQ) after the second operation.

(2) Adverse events.

\section{Surgical procedure for MSFA and implant placement}

MSFA was performed via a lateral approach when the height from the alveolar bone ridge to the maxillary sinus floor (alveolar bone height) was less than $6 \mathrm{~mm}$ at the implant placement position. When the height was more than $6 \mathrm{~mm}$, a crestal approach was selected (Fig. 1). HAp/Col was soaked in the patient's own blood before being applied into the space for MSFA. One or two blocks of HAp/Col were used in each case. All implants were placed simultaneously with MSFA, and the torque value was assessed. The second operation was performed more than 3 months after implant placement with MSFA and the implant stability quotient (ISQ) was determined using Osstell ISQ (Osstell AB, Götheborg, Sweden). The ISQ value was measured in the mesial (distal) and buccal (palatal) directions. If the value of ISQ was lower than 65 , which was considered to indicate incomplete osseointegration, the subsequent impression procedure was postponed.

\section{CBCT examination}

The alveolar bone height (Fig. 1) and cortical bone thickness of the alveolar bone ridge (cortical bone thickness) at the implant placement positions were measured on preoperative CBCT (3D Accuitomo F17D, Morita, Kyoto, Japan) images, based on the previous study [4]. Images were reconstructed using imaging software (Osirix MD, PIXME0, Geneva, Switzerland). One researcher measured each of 10 cases 3 times before the test to calculate the inter-observer error. The range of error was within $0.05 \mathrm{~mm}$. Adverse events, including symptoms of infection, were assessed during and after surgery.

\section{Results}

\section{Patients and implants}

Nineteen implants were placed into 14 maxillary sinuses. Two implants were placed in 2 maxillary sinuses via the crestal approach, and 17 implants (OsseoSpeed; 10 implants, Finesia; 1 implant, Straumann; 6 implants) were placed in 12 maxillary sinuses via the lateral approach. The second operation was performed 5.2 \pm 1.6 (range; $3-8$ ) months after the first one. The final restoration failed for 3 of the 19 implants, yielding a success rate of $84.2 \%$ (16/19 implants) (Fig. 2). All three failures occurred when the 


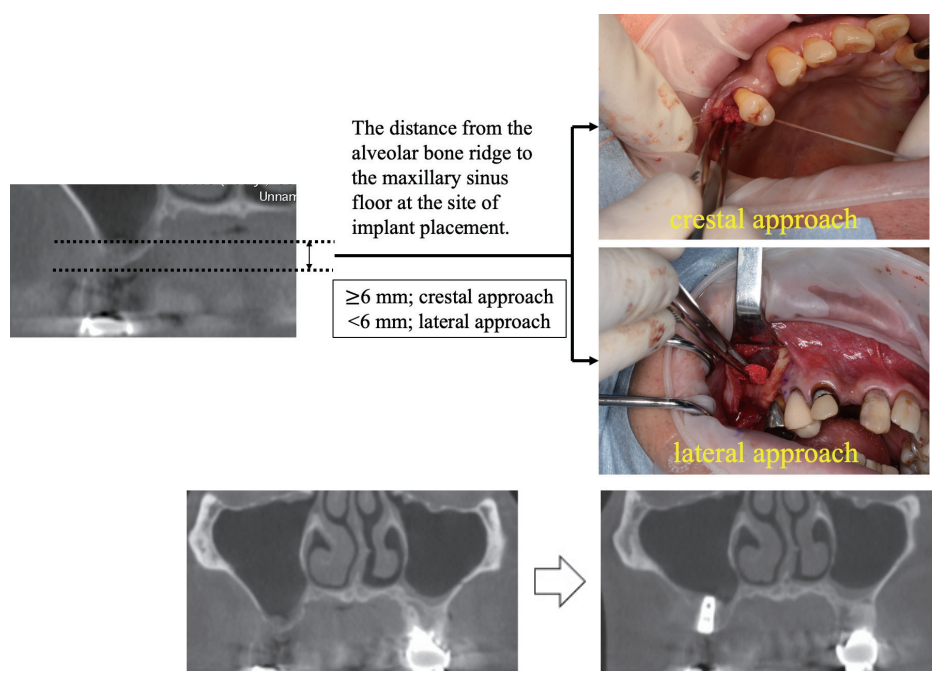

Fig. 1 Protocol used in procedures for maxillary sinus floor augmentation. When the distance from the alveolar bone ridge to the maxillary sinus floor at the site of implant placement (alveolar bone height) was more than $6 \mathrm{~mm}$, the crestal approach was selected for maxillary sinus floor augmentation. When several implants were placed, the lateral approach was selected if some locations had an alveolar bone height of less than $6 \mathrm{~mm}$.

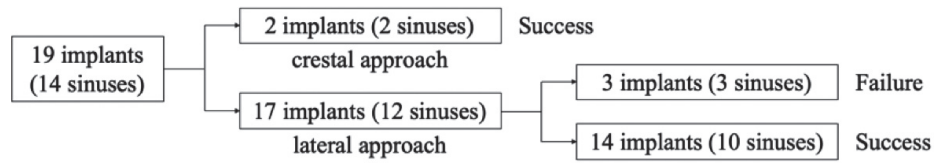

Fig. 2 Successful and failed implants inserted into maxillary sinuses. Nineteen implants were inserted into 14 maxillary sinuses. Three Fig. 2 Successful and failed implants inserted
implants failed in the lateral approach group.
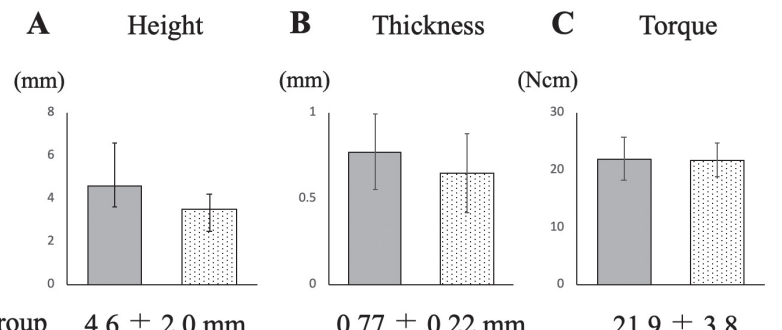

C

Torque

D

ISQ

Success group $4.6 \pm 2.0 \mathrm{~mm}$

$0.77 \pm 0.22 \mathrm{~mm}$

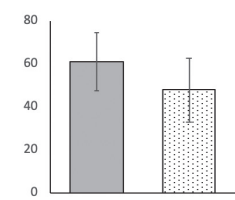

Failure group $\quad 3.5 \pm 0.7 \mathrm{~mm}$

$0.65 \pm 0.23 \mathrm{~mm}$

$21.9 \pm 3.8$

$61.0 \pm 13.3$

$21.7 \pm 2.9$

$47.8 \pm 14.8$

Fig. 3 Comparison between successful and failed implants. A: alveolar bone height, B: thickness of the alveolar cortical bone, C: value of the torque for implant placement, and D: ISQ value in the second operation. Although no significant difference was observed between the successful and failed groups, the values of A, B, and D in the failed group were lower than those in the successful group.

lateral approach was used.

\section{Lateral approach group (Figure 3)}

For the successful and failed implants, the periods required for healing from the first operation to the second were $5.2 \pm 1.7$ months and $5.2 \pm 1.0$ months, respectively (Fig. 3).

A) In the successful and failed groups, the alveolar bone heights were $4.6 \pm 2.0 \mathrm{~mm}$ and $3.5 \pm 0.7 \mathrm{~mm}$, respectively.

B) In the successful and failed groups, the cortical bone thicknesses were $0.77 \pm 0.22 \mathrm{~mm}$ and $0.65 \pm 0.23 \mathrm{~mm}$, respectively.

C) In the successful and failed groups, the implant torque values were $21.9 \pm 3.8 \mathrm{Ncm}$ and $21.7 \pm 2.9 \mathrm{Ncm}$, respectively.

D) In the successful and failed groups, the ISQ values were $61.0 \pm 13.3$ and $47.6 \pm 12.6$, respectively.

Although there were no significant differences in these 4 parameters between the successful and failed groups, the implant torque values tended to be lower in the failed group.

Details of the three failed cases (Table 1)

Case 1. A 59-year-old male who had severe habitual bruxism. An implant was placed at position 26 with the use of MSFA. The ISQ value was 59/61 at the second operation, performed 5.5 months after the first one. No untoward event was observed until the provisional restoration. The implant was rotated without any infection symptoms when the abutment was set at $35 \mathrm{Ncm}$ in the second month after the provisional restoration. MSFA with the same material and an implant placement were then performed. The ISQ value was 79/79 at the second operation, 6 months after the implant replacement. The final restoration was set 8 months after provisional restoration with adjustment of the occlusal splint for severe bruxism.

Case 2. A 69-year-old male who was receiving treatment for diabetes mellitus (HbAlc; 6.7) and rheumatoid arthritis with anti-interleukin 6 (IL-6) monoclonal antibody. The ISQ value was $35 / 35$ at the second operation, 4 months after implant placement at position 26. The implant was removed because it had rotated while setting the healing abutment without any symptoms of infection. A wider implant was placed again, and the ISQ value was $63 / 60$ at the second operation, 6 months after implant replacement. The final restoration was set 4 months after provisional restoration. Case 3. A 65-year-old female who had no notable systemic diseases. Three implants (at positions 25, 26, and 27) were placed in the maxillary sinus, and the middle one rotated in the second operation, 6 months after the first, without any symptoms of infection. At this time the ISQ values were $65 / 59$ at position 25 and $63 / 57$ at position 27 . 
Table 1 Details of the three cases of failure

\begin{tabular}{|c|c|c|c|c|c|c|c|c|c|c|}
\hline Case & Age & Gender & Smoking & Region & Implant & $\begin{array}{c}\text { Implant size } \\
(\mathrm{mm})\end{array}$ & $\begin{array}{c}\text { Height } \\
(\mathrm{mm})\end{array}$ & $\begin{array}{l}\text { Thickness } \\
(\mathrm{mm})\end{array}$ & $\begin{array}{c}\text { Healing period } \\
\text { (months) }\end{array}$ & ISQ \\
\hline 1 & 59 & M & No & 26 & FINESIA & $4.7 \times 10$ & 2.8 & 0.43 & 5.5 & $59 / 62$ \\
\hline 2 & 69 & M & No & 26 & OsseoSpeed & $5.0 \times 10$ & 3.5 & 0.63 & 4.0 & $35 / 35$ \\
\hline 3 & 65 & F & No & 26 & OsseoSpeed & $4.5 \times 11$ & 4.2 & 0.89 & 6.0 & -* \\
\hline
\end{tabular}

from the first operation to the second operation. ISQ: mesial/distal side and buccal/palatal side. *ISQ value could not be measured because the implant was rotated during removal of its cover screw.

\section{Discussion}

The implant survival rate in this study was $82.4 \%$, in comparison with $86.7-90.9 \%$ reported previously for MSFA using the lateral approach $[5,6]$. No adverse events occurred, suggesting that $\mathrm{HAp} / \mathrm{Col}$ can be used safely. However, since the number of cases examined was small, further cases should be studied to determine the applicability of HAp/Col for MSFA.

The most specific characteristic of HAp/Col is its sponge-like form, in contrast to most commercialized graft materials, which are granular. The material can be pinched with forceps and thus easily crammed into any available space for MSFA (Fig. 1). Granular material, on the other hand, needs to be scooped and is sometimes scattered around the wound, potentially causing postoperative infection. In addition, distribution of granules within the available space takes longer than is the case for HAp/ Col. Damage to the maxillary sinus membrane and infection are critical factors affecting the success of MSFA. The soft, sponge-like nature of $\mathrm{HAp} / \mathrm{Col}$ makes it less likely that tearing of the sinus membrane will occur [2]. In a study of MSFA, Barbato et al. [6] found that infection occurred in $35 \%$ of maxillary sinuses, and such infection was not controlled in $7 \%$. In the present study, no symptoms of infection were observed, suggesting that the use of $\mathrm{HAp} / \mathrm{Col}$ has a lower risk of postoperative infection. Moreover, in view of its appropriate pore size for cell migration, $\mathrm{HAp} / \mathrm{Col}$ encourages the formation of mature bone [2]. These characteristics make $\mathrm{HAp} / \mathrm{Col}$ suitable for MSFA.

López et al. [7] showed that the ISQ (degree of osseointegration) value was 63.4 in the 10th week after implant placement. In the present study, the ISQ in failed case 2 was $35 / 35$ and that in failed case 3 was not even measurable, suggesting a lack of osseointegration in these cases. Although failed case 1 showed a reasonable ISQ (59/62), an abnormal bite force might have caused the implant to fail, as the patient had severe bruxism, which is one of the causes of poor outcome after dental implant treatment. Nolan et al. [8] reported that the survival rate of implants was $73.7 \%$ when the alveolar bone height was less than $3 \mathrm{~mm}$. In addition, cortical bone thickness of the alveolar bone ridge also contributes to outcome. In failed case 1, the alveolar bone height was $2.8 \mathrm{~mm}(<3.0 \mathrm{~mm})$, and the cortical bone thickness $(0.43 \mathrm{~mm})$ was less than that in the successful group $(0.77$ $\mathrm{mm})$.

In failed case 2 the ISQ was $35 / 35$ in the second operation, 4 months after implant placement, and at 6 months after the replacement the ISQ was $63 / 60$ without additional bone augmentation. The patient had been receiving an anti-IL-6 monoclonal antibody for treatment of rheumatoid arthritis (RA). As autoimmune diseases including RA decrease bone remodeling activity [9], a prolonged period for osseointegration was possible in this case. Furthermore, the anti-IL- 6 antibody might have suppressed bone remodeling as it inhibits osteoclastogenesis via suppression of Th17 cells. Therefore, in such a case, healing might have taken longer even if HAp/ Col had encouraged early bone formation [4].

The dislodged implant in failed case 3 was located between two other adjacent implants at the time MSFA. The ISQs of the adjacent implants were $65 / 59$ and $63 / 57$, indicating sufficient osseointegration. It is possible that the area occupied by the middle implant might have stayed empty due to the high water absorption ability of $\mathrm{HAp} / \mathrm{Col}$, and that blood might have been completely absorbed by $\mathrm{HAp} / \mathrm{Col}$ in the maxillary sinus. Thus the middle implant may not have been in contact with not only the HAp/Col but also blood in the sinus.

In conclusion, $\mathrm{HAp} / \mathrm{Col}$ appears to be an appropriate material for MSFA in view of its low risk of infection and good handleability. However, alveolar bone height and cortical bone thickness may influence the success rate of MSFA using HAp/Col. The main limitation of this study was its small sample size. Therefore, further research should be conducted to assess whether this material can provide a reliable outcome in MSFA. Some minor modifications, such as increasing the volume of the filled material and soaking a sufficient amount of normal saline or peripheral blood into the material within the cavity, should be examined.

With optimization of the alveolar bone height, cortical bone thickness, and healing period, HAp/Col can be a useful graft material for MSFA with a low risk of infection.

\section{Acknowledgments}

The authors appreciate the support of Dr. Eiji Mitate, Department of Regenerative of Oral Surgery at Nagasaki University, with statistical analysis in this study and several others.

\section{Conflict of interest}

All of the hydroxyapatite/collagen composite (ReFit) used in this study was provided by HOYA Technosurgical (Tokyo, Japan).

\section{References}

1. Jambhekar S, Kernen F, Bidra AS (2015) Clinical and histologic outcomes of socket grafting after flapless tooth extraction: a systematic review for randomized controlled clinical trials. J Prosthet Dent 113, 371-382.

2. Sotome S, Orii H, Kikuchi M, Ikoma T, Ishida A, Tanaka M et al. (2006) In vivo evaluation of porous hydroxyapatite/collagen composite as a carrier of OP-1 in a rabbit OLF model. Key Eng Mater 309-311, 977-980.

3. Ohba S, Sumita Y, Nagakatani Y, Noda S, Asahina I (2019) Alveolar bone preservation by a hydroxyapatite/collagen composite material after tooth extraction. Clin Oral Investig 23, 2413-2419.

4. Sadek MM, Sabet NE, Hassan T (2016) Three-dimensional mapping of cortical bone thickness in subjects with different vertical facial dimensions. Prog Orthodont 17, 32.

5. Yoon WJ, Jeong KI, You JS, Oh JS, Kim SG (2014) Survival rate of Astra Tech implants with maxillary sinus lift. J Korean Assoc Oral Maxillofac Surg 40, 17-20.

6. Barboto L, Baldi N, Gonnelli A, Duvina M, Nieri M, Tonelli P (2018) Association of smoking habits and height of residual bone on implant survival and success rate in lateral sinus lift: a retrospective study. J Oral Imp 44, 432-438.

7. López AB, Martínez JB, Pelayo JL, García CC, Diago MP (2008) Resonance frequency analysis of dental implant stability during the healing period. Med Oral Pathol Oral Cir Buccal 13, e244-e247.

8. Nolan PJ, Freeman K, Kraut RA (2014) Correlation between Schneiderian membrane perforation and sinus lift graft outcome: a retrospective evaluation of 359 augmented sinus. J Oral Maxillofac Surg 72, 47-52.

9. Mödinger Y, Löffler B, Huber-Lang M, Ignatius A (2018) Complement involvement in bone homeostasis and bone disorders. Semin Immunol 37, 53-65. 